OESOPHAGUS

\title{
Accuracy of staging in early oesophageal cancer using high resolution endoscopy and high resolution endosonography: a comparative, prospective, and blinded trial
}

\author{
A May, E Günter, F Roth, L Gossner, M Stolte, M Vieth, C Ell
}

Gut 2004;53:634-640. doi: 10.1136/gut.2003.029421

See end of article for authors' affiliations

Correspondence to: Dr A May, Department of Internal Medicine II, HSK Wiesbaden (Teaching Hospital of Johannes Gutenberg University, Mainz), Ludwig-ErhardStrasse 100, 65199

Wiesbaden, Germany ADinahMay@aol.com

Accepted

12 November 2003

\begin{abstract}
Background and aims: The increasing use of endoscopic resection for curative treatment of early oesophageal cancers requires accurate staging before therapy. In a prospective blinded trial, we compared staging of early oesophageal carcinoma using high resolution endoscopy (HR-E) with staging using high resolution endosonography (HR-EUS).

Patients and methods: A total of 100 patients $(89$ men, 11 women; mean age 63.9 (10.8) years (range $31-91)$ ) with a suspicion of early oesophageal adenocarcinoma $(n=81)$ or squamous cell carcinoma $(n=19)$ were enrolled in the study. After endoscopic staging with high resolution video endoscopy by two experienced endoscopists, HR-EUS was performed by an experienced endosonographer who was blinded to the endoscopic assessment. Results of the staging examinations were correlated with the histology of the resected tumours.

Results: Overall rates for accuracy of the endoscopic and endosonographic staging were $83.4 \%$ and $79.6 \%$, respectively. Sensitivity for mucosal tumours ( $n=68$ ) was more than $90 \%$ (EUS $91.2 \%$, endoscopy $94.1 \%)$ while sensitivity for submucosal tumours $(n=25)$ was lower, at $48 \%$ for EUS and $56 \%$ for endoscopic staging. A combination of the two techniques increased the sensitivity for submucosal tumours to $60 \%$. Submucosal tumours in the tubular oesophagus were significantly better staged with HR-EUS than submucosal tumours close to the oesophagogastric junction $(10 / 11 v 2 / 14 ; p<0.001)$. Tumours infiltrating the second and third submucosal layers were also more correctly diagnosed than tumours with slight infiltration of the first submucosal layer (sm 1).

Conclusions: The overall diagnostic accuracy of both HR-E and HR-EUS with a $20 \mathrm{MHz}$ miniprobe in early oesophageal cancer was high (approximately $80 \%$ ), with no significant differences between the two techniques. HR-E and HR-EUS provide a high level of diagnostic accuracy for mucosal tumours and submucosal tumours located in the tubular part of the oesophagus. With submucosal tumours located at the oesophagogastric junction or with infiltration of the first third of the submucosa however, the diagnostic accuracy of both techniques is not yet satisfactory.
\end{abstract}

$\mathrm{E}$ ndoscopic treatment or resection for early malignant lesions in the oesophagus has been growing in significance in the Western world in recent years. In view of the inevitable morbidity and mortality associated with radical oesophageal resection, ${ }^{1-4}$ endoscopists have met the challenge of developing a minimally invasive-that is, endoscopictreatment procedure, and have been applying it successfully in recent years. ${ }^{5-11}$ The principal requirement for this was further development and improvement of diagnosis using high resolution video endoscopy (HR-E) and high resolution endoscopic ultrasonography (HR-EUS) devices. HR-E has made earlier and better detection of premalignant and early malignant lesions possible. It was expected that HR-EUS using the miniprobe $(20 \mathrm{MHz})$ would allow more precise assessment of the depth of tumour infiltration, making it possible to distinguish between mucosal and submucosal carcinomas. Using $7.5 \mathrm{MHz}$ endosonography (EUS), an overall accuracy of approximately $75 \%$ can be expected in assessing the $\mathrm{T}$ stage. ${ }^{12}{ }^{13}$ Advanced T3/T4 carcinomas are distinguishable from T1/T2 carcinomas in more than $80 \%$ of cases; however, adequate differentiation between $\mathrm{T} 1$ and T2 tumours, and within $\mathrm{Tl}$ tumours between mucosal and submucosal involvement was not possible. ${ }^{14}{ }^{15}$ Initial examination results from Japan using a $20 \mathrm{MHz}$ probe to assess early oesophageal carcinoma showed an accuracy of $94 \%$ for distinguishing between mucosal tumours and submucosal tumours. ${ }^{16}$ Other publications (using both $20 \mathrm{MHz}$ and
$15 \mathrm{MHz}$ probes) from Asia have reported correct staging of approximately $70-90 \%$ in early gastric and oesophageal carcinoma $^{17-20}$ and colorectal carcinoma. ${ }^{21}$

In view of further technological developments in video endoscopy using HR-E with chromoendoscopy, the question also arises of whether appropriate experience with early carcinoma on the part of the endoscopist may also provide adequate accuracy, based on endoscopic assessment alone, with regard to local endoscopic resectability with curative intent. While a prospective study comparing endoscopy and EUS in early gastric carcinoma did not demonstrate any statistically significant differences between the two techniques, EUS-with an accuracy of $71 \%$-showed a trend towards superiority over endoscopy (63\% accuracy). ${ }^{22}$ We conducted a prospective blinded study to compare endoscopy with $20 \mathrm{MHz}$ in 100 patients with early oesophageal carcinoma, as no study has previously been published on this topic in relation to oesophageal carcinoma.

\section{PATIENTS AND METHODS}

Patients

From November 1998 to July 2001, 100 patients (89 men, 11 women; mean age 63.9 (10.8) years (range 31-91)) with a suspicion of early oesophageal adenocarcinoma $(n=81)$ or

Abbreviations: EUS, endosonography; HR-E, high resolution endoscopy; HR-EUS, high resolution endosonography 
squamous cell carcinoma $(n=19)$ were enrolled into this prospective blinded comparison. Staging procedures (video endoscopy with chromoendoscopy, ultrasound of the abdomen, and computed tomography of the chest and upper abdomen) were selected in the same way as in our prospective studies on endoscopic therapy in early Barrett's adenocarcinoma and intraepithelial high grade neoplasia. ${ }^{9-11}$ After the staging procedures had been completed, a decision was taken as to whether the patient was suitable for local endoscopic resection with curative intent. Endoscopic resection was carried out using the suck and cut technique in the majority of cases. The resection techniques have been described in detail previously and can be referred to in the literature. ${ }^{9-1123}$

Exclusion criteria for endoscopic resection were any suspicion of a tumour stage more than 1 or suspicious mediastinal lymph nodes (with enlargement $>10 \mathrm{~mm}$ and/or a hypoechoic pattern). All patients with exclusion criteria were scheduled for radical oesophagectomy if they were generally operable, or for palliative endoscopic treatment if they were inoperable. If there was a suspicion of submucosal tumour infiltration, the patient received extensive information concerning surgical oesophageal resection and endoscopic therapy, including endoscopic resection, photodynamic therapy, or thermal destruction. The decision regarding further therapeutic management was made in consultation with the patient, weighing up personal risks (accompanying diseases, age, etc). All patients provided written consent to endoscopic resection. Further exclusion criteria for the trial comparing endoscopy and were: presence of low grade or high grade intraepithelial neoplasias, as well as clearly advanced tumours; endoscopic evidence of a very large and/or multifocal early cancer, for which photodynamic therapy was planned from the outset; and if blinding of the examiners was not maintained.

Analysis of the data was carried out by two team members who were not involved in collecting the endoscopic or endosonographic data (AM, FR).

\section{Endoscopic staging}

Initially, endoscopic staging was carried out by two experienced endoscopists (CE, LG) using Fujinon EG-450HR and EG-410HR high resolution endoscopes (Fujinon Europe, Inc., Willich, Germany). In addition, chromoendoscopy with a special spraying catheter (Olympus PW-5L; Olympus, Inc., Hamburg, Germany) was carried out in all patients, using methylene blue $0.5 \%$ in cases of Barrett's oesophagus and Lugol's solution $1 \%$ in cases of squamous cell cancer. After endoscopy, a standardised questionnaire was completed in which the endoscopist stated his assessment regarding the location of the tumour (oesophagogastric junction, distal, middle, or upper part of the oesophagus), its macroscopic classification according to the Japanese classification ${ }^{24}$ of
Table 2 Endosonographic (EUS) and endoscopic staging of early carcinomas $(n=94)$

\begin{tabular}{|c|c|c|c|}
\hline \multirow[b]{2}{*}{ Early oesophageal cancers } & \multicolumn{3}{|c|}{ Patients } \\
\hline & $\mathbf{n}$ & Total & $\%$ \\
\hline \multicolumn{4}{|l|}{ All cancers $(n=94)$} \\
\hline EUS correct & 75 & 94 & 79.8 \\
\hline Endoscopy correct & 78 & 94 & 82.9 \\
\hline EUS understaging & 13 & 94 & 13.8 \\
\hline Endoscopic understaging & 10 & 94 & 10.6 \\
\hline EUS overstaging & 6 & 94 & 6.4 \\
\hline Endoscopic overstaging & 6 & 94 & 6.4 \\
\hline \multicolumn{4}{|l|}{ Mucosal cancers $(n=68)$} \\
\hline EUS correct & 62 & 68 & 91.2 \\
\hline Endoscopy correct & 64 & 68 & 94.1 \\
\hline EUS overstaging & 6 & 68 & 8.8 \\
\hline Endoscopic overstaging & 4 & 68 & 5.9 \\
\hline \multicolumn{4}{|l|}{ Submucosal cancers $(n=25)$} \\
\hline EUS correct & 12 & 25 & 48.0 \\
\hline Endoscopy correct & 14 & 25 & 56.0 \\
\hline EUS understaging & 13 & 25 & 52.0 \\
\hline Endoscopic understaging & 10 & 25 & 40.0 \\
\hline EUS overstaging & 0 & 25 & 0 \\
\hline Endoscopic overstaging & 2 & 25 & 8.0 \\
\hline
\end{tabular}

early carcinomas (type I, polypoid; type IIa, elevated; IIb, flat; IIc, depressed; or type III, excavated) and the combination of these types (for example, type IIa+b implies that the tumour has elevated and flat parts; type IIa+c that the tumour has elevated and depressed parts; type IIb+c that the tumour has flat and depressed parts; and type I+IIa that the tumour has a polypoid and an elevated part), as well as the depth of tumour infiltration (mucosal or submucosal). Polypoid, depressed, and excavated configurations and ulcerations were regarded as endoscopic signs consistent with a higher rate of submucosal infiltration.

\section{Endosonographic staging}

The next day, HR-EUS using a $20 \mathrm{MHz}$ miniprobe (Fujinon SP-501; Fujinon Europe, Inc.) as a "through the scope" probe with double channel video endoscopes (Fuji ED 410 D double channel; Fujinon Europe, Inc.) was carried out by an experienced endosonographer (EG). Approximately 100$200 \mathrm{ml}$ water volumes were instilled through the second channel of the endoscope using a pump system (Olympus UWS-1; Olympus, Inc.). To avoid aspiration, patients were examined with an elevated position of the upper part of the body. The endosonographer was blinded to the results of the endoscopic evaluation, and his assessment of the depth of tumour invasion (mucosal/submucosal) was also recorded using a standardised questionnaire. In addition, conventional was carried out (Olympus GF-UM-20, 7.5 MHz, Olympus, Inc.) in order to assess lymph node status.

Table 1 Macroscopic classification by video endoscopy $(n=94)^{*}$

\begin{tabular}{lclll}
\hline Macroscopic type $\left(\right.$ Nishi $\left.^{24}\right)$ & AdenoCa $(\mathbf{n}=\mathbf{7 7})^{*}$ & $\mathbf{S C C}(\mathbf{n}=\mathbf{1 7})^{*}$ & Total $(\mathbf{n}=\mathbf{9 4})$ & sm infiltration $(\mathbf{n}=\mathbf{2 5})$ \\
\hline Type I & 9 & 2 & 11 & $7 / 11$ \\
Type lla & 26 & 2 & 28 & $4 / 28$ \\
Type Ilb & 16 & 4 & 20 & $1 / 20$ \\
Type Ilc & 2 & 1 & 3 & $2 / 3$ \\
Type III & 4 & 1 & 5 & $1 / 5$ \\
Type Ila+b & 8 & 1 & 9 & $0 / 9$ \\
Type Ila+c & 15 & 7 & 22 & $9 / 22$ \\
Type Ilb+c & 1 & 0 & 1 & $0 / 1$ \\
Type I+lla & 1 & 1 & 2 & $1 / 2$ \\
\hline
\end{tabular}

*Six patients were excluded due to missing histology (four in the adenocarcinoma group and two in the SCC group).

AdenoCa, adenocarcinoma; SCC, squamous cell carcinoma; sm, submucosa. 
Table 3 Correct staging of submucosal carcinomas by high resolution endoscopic ultrasonography (HR-EUS) and HR-endoscopy (HR-E) in relation to the different submucosal types (sm1, sm2, and sm3)

\begin{tabular}{|c|c|c|c|c|c|}
\hline \multirow[b]{2}{*}{ Submucosal type } & \multicolumn{2}{|l|}{ HR-EUS } & \multicolumn{2}{|l|}{ HR-E } & \multirow[b]{2}{*}{ Total $(n=25$} \\
\hline & Correct & Not correct & Correct & Not correct & \\
\hline sml & 4 & 8 & 5 & 7 & 12 \\
\hline sm2 & 2 & 2 & 3 & 1 & 4 \\
\hline sm3 & 6 & 3 & 6 & 3 & 9 \\
\hline Total & 12 & 13 & 14 & 11 & 25 \\
\hline
\end{tabular}

sm1, first submucosal layer; sm2, second submucosal layer; sm3, third submucosal layer.

All endoscopies and endosonographies were carried out with the patient under analgesic sedation (midazolam 1$10 \mathrm{mg}$ and/or pethidine $25-100 \mathrm{mg}$ intravenously), with continuous monitoring of heart rate and oxygen saturation.

\section{Histology}

The gold standard used to establish the accuracy of endoscopic and endosonographic staging examinations was histological examination of the endoscopically or surgically resected tumour. The histological criteria used for early oesophageal carcinoma were the WHO criteria and the Vienna classification. ${ }^{25}{ }^{26}$ Generally, the resected specimens were fixed in $4 \%$ formalin, dehydrated in gradual alcohol series, and embedded in paraffin. Serial step sections of $4 \mu \mathrm{m}$ were cut and stained with haematoxylin and eosin and/or alcian blue, $\mathrm{pH} 2.5$.

The histological assessment was carried out by experienced pathologists (MS, MV). After resection, specimens were examined with regard to tumour depth (involvement of the mucosa or submucosa) - the principal point of this study. Other histological assessment criteria were: freedom from tumour at the base and lateral margins of the specimens; degree of differentiation (grade G1, well differentiated; G2, moderately differentiated; G3, poorly differentiated); and in some cases invasion of the lymphatic vessels and veins.

\section{Statistical evaluation}

Descriptive statistics were calculated for patient data and clinical parameters: mean, median, SD, and range (minimum-maximum). Paired proportions may also be shown as a contingency table. Although the table closely resembles those relating to comparison of two independent proportions, it is essential to remember that the proportions are paired and so the usual $\chi^{2}$ test is inappropriate.

Comparison of paired proportions is based on the frequencies of pairs with different outcomes. The value of the test statistic is referred to the $\chi^{2}$ distribution with one degree of freedom. The test of paired proportions is often known as McNemar's test, especially when the data are shown as $2 \times 2$ contingency table. Generalization for $k \times k$ tables is stated as "test of symmetry".

Table 4 Accuracy of high resolution endoscopic ultrasonography (HR-EUS) and HR-endoscopy (HR-E) in early oesophageal carcinomas

\begin{tabular}{llll}
\hline & \multicolumn{2}{l}{$\begin{array}{l}\text { Proportion of correct } \\
\text { results }\end{array}$} & \\
\cline { 2 - 3 } & $\mathbf{m}$ & sm & Accuracy \\
\hline HR-E & 64 & 14 & $78 / 93^{*}=83.4 \%$ \\
HR-EUS & 62 & 12 & $74 / 93^{*}=79.6 \%$ \\
\hline
\end{tabular}

*94 patients were enrolled in the study, one patients showed an T2carcinoma, therefore we have 93 early cancers.

$\mathrm{m}$, mucosa; sm, submucosal layer.
In addition, a kappa statistic was performed to test for agreement of the diagnostic methods. Here, a kappa value below 0.4 was regarded as representing poor agreement, a kappa value of $0.41-0.60$ fair agreement, a kappa value of $0.61-0.80$ good agreement, and a kappa value greater than 0.80 excellent agreement.

Statistical tests were performed using SAS software, version 8.2 (Cary, North Carolina, USA).

\section{Ethical considerations}

All patients provided written consent to undergo local therapy after receiving extensive information. All patients were informed that surgery was the current treatment of choice for early oesophageal carcinoma, and received a brochure describing endoscopic resection as an experimental

Table 5 Correct staging of submucosal carcinomas by endoscopic ultrasonography (HR-EUS) and endoscopy (HR-E) in relation to the different locations of the carcinoma (oesophagogastric junction $v$ tubular oesophagus)

\begin{tabular}{llll}
\hline & \multicolumn{2}{l}{ Histology } & \\
\cline { 2 - 3 } & $\mathbf{M}$ & $\mathbf{s m}$ & Total \\
\hline $\begin{array}{l}\text { Oesophagogastric junction } \\
\text { HR-EUS/HR-E }\end{array}$ & & & \\
$\quad \mathrm{m}$ & $33 / 34$ & $12 / 8$ & 45 \\
$\quad$ sm & $4 / 3$ & $2 / 5(+1)^{*}$ & 6 \\
$\quad$ Total & $37 / 37$ & $14 / 14$ & 51 \\
Tubular oesophagus & & & \\
$\quad$ HR-EUS/HR-E & & & \\
$\quad \mathrm{m}$ & $29 / 30$ & $1 / 1$ & 30 \\
$\quad$ sm & $2 / 1$ & $10 / 9(+1)^{*}$ & 12 \\
Total & 31 & 11 & 42 \\
\hline
\end{tabular}

*One sm carcinoma was assessed as a T2 tumour by HR-E. $\mathrm{m}$, mucosa; sm, submucosal layer.

Table 6 Correct staging of submucosal carcinomas by endoscopic ultrasonography (HR-EUS) and endoscopy $(H R-E)$ in relation to the different locations of the carcinoma loesophagogastric junction $v$ tubular oesophagus) and kappa values

\begin{tabular}{|c|c|c|}
\hline Kappa value & $95 \% \mathrm{Cl}$ & p Value \\
\hline \multicolumn{3}{|c|}{ Oesophagogastric junction } \\
\hline $\begin{array}{l}\text { HR-EUS/HR-E } \\
0.042 / 0.346\end{array}$ & $\begin{array}{c}\text { HR-EUS/HR-E } \\
-0.211-0.296 / \\
0.072-0.619\end{array}$ & $\begin{array}{l}\text { HR-EUS/HR-E } \\
0.262 / 0.352\end{array}$ \\
\hline \multicolumn{3}{|l|}{ Other locations } \\
\hline $0.836 / 0.776$ & $\begin{array}{l}0.656-1.016 / \\
0.584-0.967\end{array}$ & $0.954 / 1.000$ \\
\hline $\begin{array}{l}\text { Test for equal kappa } \\
\text { coefficients }\end{array}$ & & $p<0.001 / p=0.012$ \\
\hline
\end{tabular}

$95 \% \mathrm{Cl}, 95 \%$ confidence interval. 

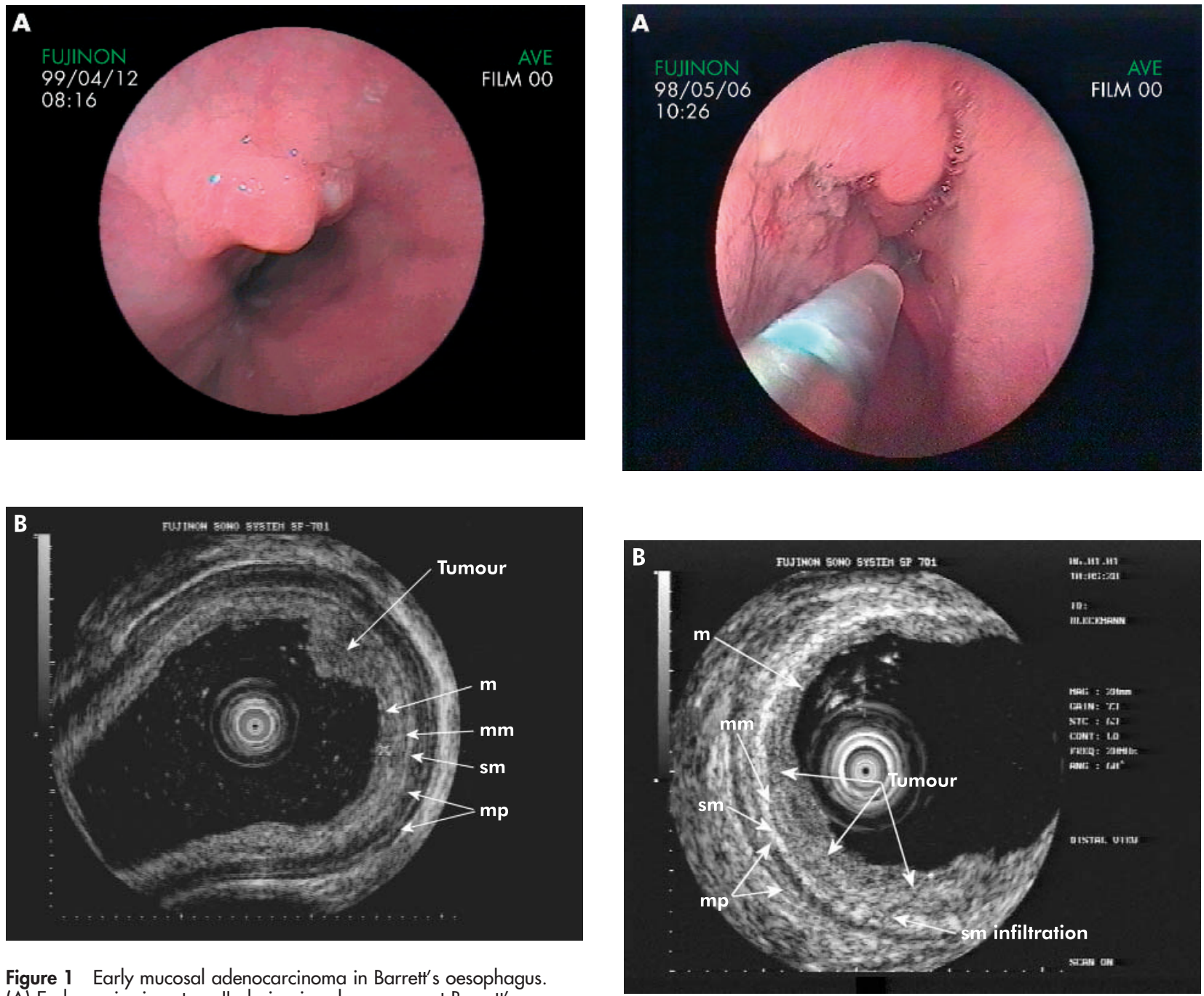

Figure 1 Early mucosal adenocarcinoma in Barrett's oesophagus. (A) Endoscopic view: type lla lesion in a long segment Barrett's oesophagus. (B) High resolution endoscopic ultrasonography: the elevated mucosal tumour corresponds to a circumscript thickening of the mucosa. m, mucosa, mm, muscularis mucosae; sm, submucosa; mp, muscularis propria.

treatment. If patients were interested in obtaining a second opinion, surgeons were regularly asked to discuss all questions once again with the patients.

Approval for the study was received from the ethics committee of the State of Hesse, Germany, in accordance with good clinical practice requirements.

\section{RESULTS}

Six of 100 patients had to be excluded from the study. Histological assessment was not possible in five patients with a suspicion of a large submucosal tumour as a decision was made to carry out palliative thermal destruction (APC in one of the five patients and Nd:YAG laser in the other four) due to concomitant diseases and/or age over 70 years. In one patient, carcinoma was not identified in the endoscopically resected specimen although histology of the biopsy in this area had shown a carcinoma. No cancer was found in subsequent check up endoscopies. The neoplasia had probably been so small that extensive biopsies had already removed it.

Endoscopic resection was carried out in 92 patients; five patients underwent surgical oesophageal resection. Three of these five patients were referred to surgery after pretherapeutic staging: submucosal tumour infiltration was seen in

Figure 2 Early submucosal adenocarcinoma in Barrett's oesophagus: sm1 type. (A) Endoscopic view: type lla+c lesion in the distal part of a long segment Barrett's oesophagus. (B) High resolution endoscopic ultrasonography: broad thickening of the mucosa with a small focal infiltration of the first submucosal layer. $m$, mucosa, mm, muscularis mucosae; sm, submucosa; mp, muscularis propria.

two patients, with suspicious lymph nodes in one; in the third patient, a T2 tumour was diagnosed endosonographically. In two of the five patients who underwent surgery, in whom at least one of the diagnostic procedures assessed a submucosal tumour, endoscopic resection was carried out first. Histological classification of the resected specimens not only confirmed a submucosal tumour but also revealed infiltration of the third submucosal layer $(\operatorname{sm} 3)$. These two patients therefore also underwent surgery for oesophageal resection.

Detailed results with regard to macroscopic classification and histology of the cancers are given in table 1. Comparisons of the endosonographic and endoscopic staging results are given in tables 2 and 3. The overall sensitivity rates were $79.8 \%$ for and $82.9 \%$ for endoscopy. If the two diagnostic methods were combined and submucosal infiltration was assumed if one of the methods postulated submucosal infiltration, overall sensitivity increased to $84 \%$ (79/94); correct staging of submucosal cancers increased from $48 \%$ (with alone) and 56\% (with endoscopy alone) to $60 \%$ if the two methods were combined. Submucosal infiltration was not diagnosed with either method in $40 \%$ of cases. The 

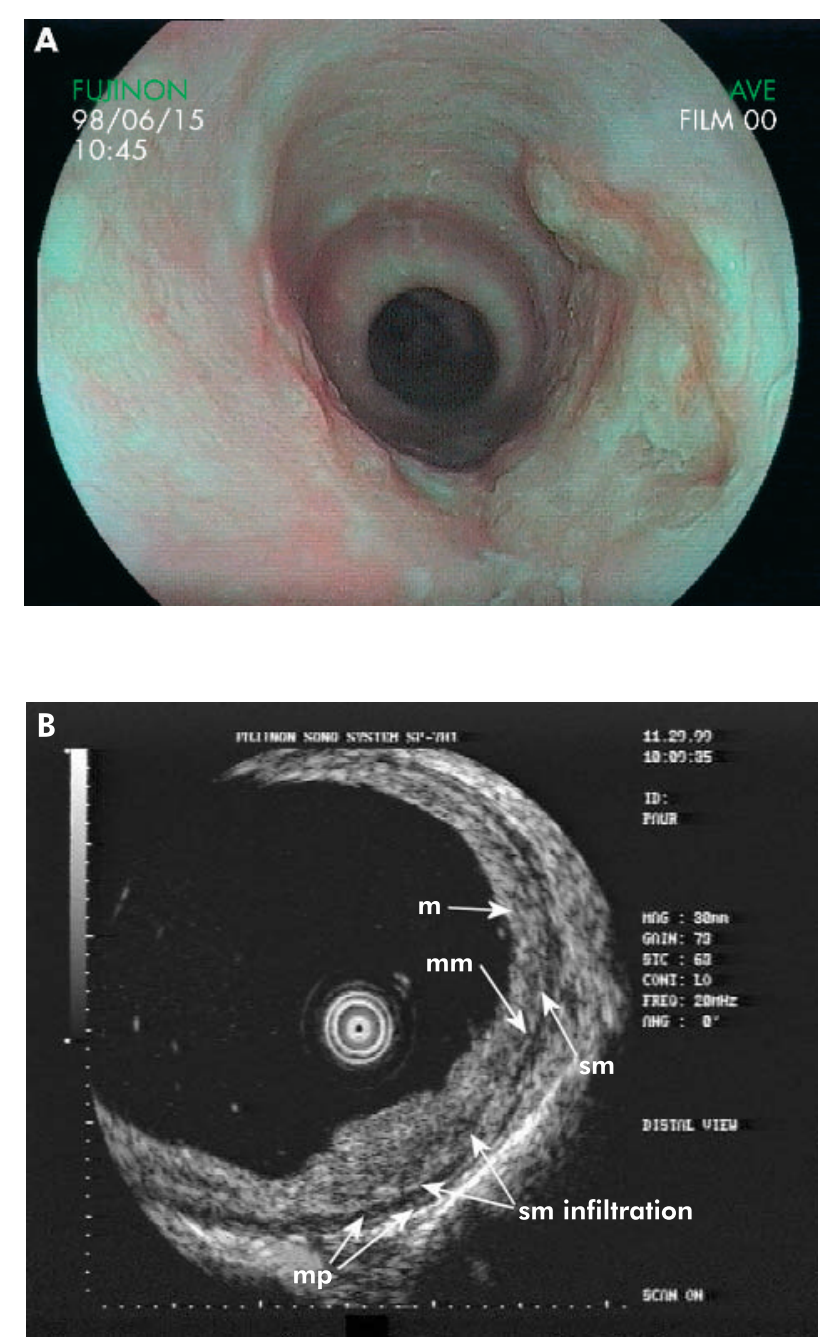

Figure 3 Early submucosal squamous cell carcinoma: sm3 type. (A) Endoscopic view: large submucosal tumour with depressed and elevated parts as well as ulcerations at the margins of the tumour. (B) High resolution endoscopic ultrasonography: broad and deep tumour infiltration of the submucosa, even extending to the third submucosal layer. $\mathrm{m}$, mucosa, mm, muscularis mucosae; sm, submucosa; $\mathrm{mp}$, muscularis propria.

accuracy of HR-E staging was $83.4 \%$ and the accuracy of HR-EUS was $79.6 \%$ (table 4 ).

Twenty five of the 94 patients $(26.6 \%)$ had histologically confirmed submucosal tumours. Two of the submucosal cancers were located in the proximal part of the oesophagus, four in the middle part, and four in the distal part, with a distance to the oesophagogastric junction of approximately $2 \mathrm{~cm}$ or more. Endosonographic staging was correct in nearly all cancers located in the tubular oesophagus (10/11; 90.9\%) whereas only two of 14 cancers ( $14.3 \%$ ) located directly at the oesophagogastric junction were correctly assessed by. This means that endosonographic evaluation of early cancers of the tubular oesophagus is significantly better statistically $(\mathrm{p}<0.001)$ in comparison with lesions at the oesophagogastric junction. Similar results were obtained for HR-E; staging was significantly better in the tubular oesophagus than at the oesophagogastric junction. Details of both methods are given in tables 5 and 6 . The same was true for submucosal infiltration of the first submucosal layer (type sml); onethird of sml cancers (4/12) were correctly diagnosed, and eight of $12 \mathrm{sml}$ cancers were not correctly assessed but a significant difference (table 3) could not be demonstrated because of the low number of cases.

With regard to the macroscopic classification, type I and type IIa+c lesions were the leading types of submucosal carcinoma: seven of 11 type I lesions (63.6\%) showed submucosal infiltration, and submucosal infiltration was found in nine of 22 type IIa+c lesions (40\%) (see figs 1-3 for examples). Submucosal infiltration was seen more rarely in type IIa lesions $(4 / 28 ; 14 \%)$. Other types were rarely associated with submucosal infiltration (1/21 type IIb lesions; $5 \%)$, or the number of cases was too small for reliable assessment to be made (2/3 type IIc lesions; 1/5 type III lesions). A significant association was not observed between macroscopic type and depth of submucosal infiltration ( $\mathrm{sml}$, $\mathrm{sm} 2$, or sm3). There was a trend towards more frequent sm3 tumours with type I lesions while in contrast sml lesions more frequently corresponded macroscopically to type IIa+c lesions. In this group of patients, type IIb lesions were only seen in sml tumours; a type IIb lesion was never seen in sm2 or sm3 tumours. Ulcerated type III lesions were never found in submucosal cancers of the sml type.

\section{DISCUSSION}

Conventional is currently the most accurate method for pretherapeutic assessment of lymph node status in oesophageal carcinoma, and is clearly superior to computed tomography and magnetic resonance imaging. In , ultrasound endoscopes with 7.5 $\mathrm{MHz}$ ultrasound probes are used either as radial or linear scanners. Conventional EUS is also currently the best diagnostic method for assessing the depth of tumour infiltration (the T category)..$^{12}$

However, conventional EUS systems are not ideal for assessing early neoplastic changes, and mucosal and submucosal carcinomas in particular, as the endoscopic view is limited and changes cannot be viewed directly. Early neoplastic lesions may also be pressed into the oesophageal wall by the filled balloon required for water preparation. In addition, conventional EUS systems use $7.5 \mathrm{MHz}$ or maximally $12 \mathrm{MHz}$ ultrasound probes, which are inferior to $20 \mathrm{MHz}$ ultrasound probes with regard to resolution of the tissue layers in the near field.

These disadvantages of conventional EUS are not found with EUS miniprobes, which are therefore suitable for differentiating wall infiltration in Tl carcinomas. Miniprobes are applied under direct vision in a targeted fashion in the area of an endoscopically detected lesion, without pressing the lesion into the oesophageal wall. Using a double channel endoscope, allowing simultaneous working processes, sufficient liquid can be instilled through the second channel for water preparation in spite of active peristalsis in the oesophagus.

Larger studies on HR-EUS published in Western journals have mainly been by Asian research groups, and they have focused on early gastric carcinoma and early neoplasms of the large bowel. ${ }^{17} 192127$ There have been only a few reports, some with small numbers of patients, on EUS and particularly HR-EUS with the miniprobe in early oesophageal cancer. ${ }^{16} 182028$ For example, Murata and colleagues ${ }^{16}$ reported correct differentiation between mucosal and submucosal carcinomas using a $20 \mathrm{MHz}$ probe in $94 \%$ of cases (46/49 lesions). The overall accuracy in the 54 patients investigated was $75 \%$. Yanai and colleagues ${ }^{18}$ found an accuracy of $72.3 \%$ in staging 47 mucosal or submucosal lesions; the overstaging rate was $19 \%$ and the understaging rate was $2 \%$.

With 100 patients, the present study is currently the largest investigation, providing a prospective assessment of the value of HR-EUS with miniprobes in early oesophageal carcinoma and comparing the results with histological findings after endoscopic resection. The endosonographic accuracy of early 
oesophageal carcinomas showed good results at approximately $80 \%$, a value comparable with that in a Japanese study of early gastric carcinoma ${ }^{17}$ and in a study of early oesophageal carcinoma ${ }^{18}$ where Yanai et al reported an accuracy of $72.3 \%$ and $64.7 \%$ ( $80 \%$ for mucosal carcinoma). Analysis of submucosal carcinomas, representing approximately one quarter of early oesophageal carcinomas, is all the more disappointing. For these lesions, sensitivity fell to $48 \%$ (with a specificity of 91\%). Two factors appear to have played a part here: location of the carcinomas and depth of infiltration. Only two of 14 carcinomas located directly at the oesophagogastric junction were correctly assessed, while nine of 10 carcinomas in the rest of the oesophagus were correctly staged. Technical difficulties due to the anatomical conditions in the area of the cardia, particularly in the presence of an axial hiatal hernia, certainly played a major role. Data from the literature confirm this: de Manzoni and colleagues ${ }^{29}$ reported an overall accuracy of $55.2 \%$ for EUS assessment of adenocarcinoma of the cardia, with $\mathrm{T} 2$ tumours showing particularly poor results. Kelly and colleagues, ${ }^{15}$ on the basis of a literature search conducted for their review article, similarly stated that the accuracy of EUS is lower for carcinomas of the cardia. It is often not possible to achieve adequate water preparation by instilling fluid through the second channel. In addition, there is substantial motility that prevents dilatation of the distal oesophagus from being maintained for a longer period. With regard to water preparation, a condom-like sheath into which water could be introduced from outside for preparation might be helpful. Initial prototypes with a total diameter of $2.6 \mathrm{~mm}$, capable of being introduced through a $3.2 \mathrm{~mm}$ instrument channel, have already been successfully tested in clinical practice. ${ }^{30}$

The second factor is the superficial — often focal-infiltration of the uppermost layer of the submucosa, known as type sml. Eight of 12 sml-type lesions were not correctly diagnosed; all were located in the distal oesophagus $(\mathrm{n}=1)$ or directly at the oesophagogastric junction $(n=7)$, again underlining the importance of tumour location. The fact that the sml type represents an independent "risk factor" has already been noted by Shimizu and colleagues. ${ }^{20}$ In addition, concomitant inflammatory changes in the oesophageal mucosa-which are often seen in reflux induced Barrett's oesophagus despite administration of proton pump inhibitors-can make the assessment very much more difficult.

As described by Shimizu and colleagues, ${ }^{20}$ overall sensitivity improves if sml carcinomas are included in the mucosal group. If it is assumed on the basis of the data presented by Gotoda and colleagues ${ }^{31}$ that sml-type cancers are endoscopically resectable with no risk, or a very low risk, of lymph node metastasis under certain conditions, patients with histologically confirmed tumour infiltration of the first submucosal layer (sml-type) could be assigned on clinical grounds to the group of patients with mucosal cancers. On this assumption, the overall correct staging of endoscopic and endographic staging would increase to $89 \%$ and $86 \%$, respectively. In the subgroup of submucosal carcinomas, the correct assessment would increase from $48 \%$ to $80 \%$. The best diagnostic yield was achieved when the two diagnostic methods were combined, as has already been postulated by Ohashi and colleagues. ${ }^{32}$ The overall rate of correct staging was then $91.5 \%(86 / 94)$, with correct assessment of submucosal carcinomas at $88 \%(22 / 25)$, provided that submucosal infiltration was assumed to be present if one of the two diagnostic procedures identified it.

On the basis of the data presented by Gotoda and colleagues $^{31}$ on early gastric carcinoma, this regrouping of sml carcinomas using clinical criteria appears to be worth considering. A follow up study of more than 5200 patients with early gastric carcinoma who had undergone surgery showed that no lymph node metastases were found when the lesion was type sml without evidence of infiltration of lymphatic vessels and a maximum size of up to $30 \mathrm{~mm}$. This means that gastric sml carcinomas can receive the same type of endoscopic treatment as mucosal carcinomas under certain conditions. Shimizu and colleagues ${ }^{33}$ recently presented long term results on this topic regarding endoscopic treatment of early squamous cell carcinomas that had infiltrated the muscularis mucosae $(\mathrm{m} 4)$ or had already penetrated the submucosa ( $\mathrm{sml}$ ). Five year survival rates (YSR) and tumour specific five YSR for patients who had received endoscopic treatment did not differ significantly from those in the control group who had undergone surgical resection. The extent to which these data might be generally applicable to early oesophageal carcinoma of Barrett's type remains an open question.

The results of endoscopic staging, which with an overall sensitivity of $82.9 \%$ and an accuracy of $83.4 \%$ are even slightly superior to those of EUS (79.8\% and $79.6 \%)$, are impressive. However, an absolute prerequisite is that the endoscopist should have a substantial amount of experience with early carcinomas and high resolution endoscopes. Endoscopy is also more sensitive than EUS in the assessment of submucosal carcinomas ( $56 \% \vee 48 \%$, respectively). These results are not entirely in agreement with Japanese data from a comparative study on early gastric carcinoma ${ }^{22}$ in which endoscopy, with an accuracy of $63 \%$, was inferior to EUS at $71 \%$. No tendency towards overstaging with EUS was found in the present study; on the contrary, submucosal carcinomas tended to be rather underestimated.

In conclusion, overall correct diagnostic assessment of both HR-E and HR-EUS with a $20 \mathrm{MHz}$ miniprobe in early oesophageal cancer is high, with no significant differences between the two techniques. Combining HR-E and HR-EUS can increase sensitivity. HR-E and HR-EUS provide a high level of diagnostic accuracy for mucosal and submucosal tumours located in the tubular part of the oesophagus. With submucosal tumours located at the oesophagogastric junction or with infiltration of the first third of the submucosa however, the diagnostic meaningfulness of both techniques is not yet satisfactory.

\section{Authors' affiliations}

A May, E Günter, F Roth, L Gossner, C Ell, Department of Medicine II, HSK Wiesbaden (Teaching Hospital of the University of Mainz),

Germany

M Stolte, Department of Pathology, Bayreuth (Teaching Hospital of the University of Erlangen), Germany

M Vieth, Department of Pathology, University of Magdeburg, Germany

\section{REFERENCES}

1 Heitmiller RF, Redmond M, Hamilton SR. Barrett's esophagus with high-grade dysplasia: an indication for prophylactic esophagectomy. Ann Surg 1996;224:66-71

2 Hölscher AH, Bollschweiler E, Schneider PM, et al. Early adenocarcinoma in Barrett's oesophagus. Br J Surg 1997;84:1470-3.

3 Nigro JJ, Hagen JA, DeMeester TR, et al. Occult esophageal adenocarcinoma: extent of disease and implications for effective therapy. Ann Surg 1999;230:433-40.

4 Stein HJ, Feith M, Mueller J, et al. Limited resection for early adenocarcinoma in Barrett's esophagus. Ann Surg 2000;232:733.

5 Gossner L, May A, Sroka R, et al. Photodynamic destruction of high grade dysplasia and early carcinoma of the esophagus after oral administration of 5-aminolevulinic acid. Cancer 1999:86:1921-8.

6 Overholt BF, Panjehpour M, Haydek JM. Photodynamic therapy for Barrett's esophagus: follow-up in 100 patients. Gastrointest Endosc 1999;49:1-7.

7 Gossner L, Stolte M, Sroka R, et al. Photodynamic ablation of high-grade dysplasia and early cancer in Barrett's esophagus by means of 5aminolevulinic acid. Gastroenterology 1998; 114:448-55.

8 Takeshita K, Tani $M$, Inove $H$, et al. Endoscopic treatment of early oesophageal or gastric cancer. Gut 1997;40:123-7.

9 Ell C, May A, Gossner L, et al. Endoscopic mucosal resection of early cancer and high grade dysplasia in Barrett's esophagus. Gastroenterology 2000;1 18:670-7. 
10 May A, Gossner L, Pech O, et al. Intraepithelial high-grade neoplasia and early adenocarcinoma in short-segment Barrett's esophagus (SSBE): curative treatment using local endoscopic treatment techniques. Endoscopy 2002;34:604-10.

11 May A, Gossner L, Pech O, et al. Local endoscopic therapy for intraepithelial high-grade neoplasia and early adenocarcinoma in Barreft's oesophagus: acute-phase and long-term results of a new treatment approach. Eur J Gastroenterol Hepatol 2002;14:1085-91.

12 Greenberg J, Durkin $M$, van Drunen $M$, et al. Computed tomography or endoscopic ultrasonography in preoperative staging of gastric and esophageal tumors. Surgery 1994;116:696-701.

13 Meining A, Dittler HJ, Wolf A, et al. You get what you expect? A critical appraisal of imaging methodology in endosonographic cancer staging. Gut 2002;50:599-603.

14 Hiele M, De Leyn P, Schurmans P, et al. Relation between endoscopic ultrasound findings and outcome of patients with tumors of the esophagus or esophagogastric junction. Gastrointest Endosc 1997;45:381-6.

15 Kelly S, Harris KM, Berry E, et al. A systematic review of the staging performance of endoscopic ultrasound in gastro-oesophageal carcinoma. Gut 2001;49:534-9.

16 Murata Y, Suzuki S, Ohta M, et al. Small ultrasonic probes for determination of the depth of superficial esophageal cancer. Gastrointest Endosc 1996:44:23-8.

17 Yanai $\mathbf{H}$, Tada M, Karita $M$, et al. Diagnostic utility of 20-megahertz linear endoscopic ultrasonography in early gastric cancer. Gastrointest Endosc 1996;44:29-33.

18 Yanai H, Yoshida T, Harada T, et al. Endoscopic ultrasonography of superficial esophageal cancers using a thin ultrasound probe system equipped with switchable radial and linear scanning modes. Gastrointest Endosc 1996;44:578-82.

19 Hasegawa N, Niwa Y, Arisawa T, et al. Preoperative staging of superficial carcinoma: comparison of a standard probe and standard endoscopic ultrasonography. Gastrointest Endosc 1996;44:388-93.

20 Shimizu Y, Tsukagoshi H, Nakazato T, et al. Clinical evaluation of endoscopic ultrasonography (EUS) in the diagnosis of superficial esophageal carcinoma. Rinsho Byori 1995;43:221-6.
21 Yanai H, Noguchi T, Mizumachi S, et al. A blind comparison of the effectiveness of endoscopic ultrasonography and endoscopy in staging early gastric cancer. Gut 1999;44:361-5.

22 Saitoh Y, Obara T, Einami K, et al. Efficacy of high-frequency ultrasound probes for the preoperative staging of invasion depth in flat and depressed colorectal tumors. Gastrointest Endosc 1996;44:34-9.

23 Ell C, May A, Wurster $\mathrm{H}$. The first reusable multiple band ligator for endoscopic hemostasis of variceal bleeding, nonvariceal bleeding and mucosal resection. Endoscopy 1999;31:1-3.

24 Nishi M, Omori Y, Miwa K. Japanese Research Society for Gastric Cancer. Japanese classification of gastric carcinoma, 1st English edn. Tokyo: Kanehara, 1995.

25 Watanabe H, Jass JR, Sobin LH. Histological typing of oesophageal and gastric tumours, 2nd edn. Berlin: Springer, 1990.

26 Schlemper RJ, Riddell RH, Kato Y, et al. The Vienna classification of gastrointestinal epithelial neoplasia. Gut 2000;47:251-5.

27 Hizawa K, Iwai K, Esaki M, et al. Is endoscopic ultrasonography indispensable in assessing the appropriateness of endoscopic resection for gastric cancer? Endoscopy 2002;34:973-8.

28 Scotiniotis IA, Kochman ML, Lewis JD, et al. Accuracy of EUS in the evaluation of Barrett's esophagus and high-grade dysplasia or intramucosal carcinoma. Gastrointest Endosc 2001;54:689-96.

29 de Manzoni G, Pedrazzani C, Di Leo A, et al. Experience of endoscopic ultrasound in staging adenocarcinoma of the cardia. Eur J Surg Oncol 1999;25:595-8.

30 Kawano T. Soft balloon method in esophageal endoscopic ultrasound with a 20-MHz ultrasound thin probe. Gastrointest Endosc 2002;56:S107 (A29)

31 Gotoda T, Yanagisawa A, Sasako M, et al. Incidence of lymph node metastasis from early gastric cancer: estimation with a large number of cases at two large centers. Gastric Cancer 2000;3:219-25.

32 Ohashi S, Segawa K, Okamura S, et al. The utility of endoscopic ultrasonography and endoscopy in the endoscopic mucosal resection of early gastric cancer. Gut 1999;45:599-604.

33 Shimizu $Y$, Tsukagoshi H, Fujita $M$, et al. Long-term outcome after endoscopic mucosal resection in patients with esophageal squamous cell carcinoma invading the muscularis mucosae or deeper. Gastrointest Endosc 2002;56:387-90. 